\title{
Non-monotonic association between chlorinated polyfluorinated ether sulfonic acids exposure and the risk of overweight/obesity status in adults
}

Chu Chu

Sun Yat-Sen University

Qiu-Ling Fang

Sun Yat-Sen University

Xin-Xin Cui

SunYat-Sen University

Zhengmin(Min) Qian

Saint Louis University

Stephen Edward McMillin

Saint Louis University

Steven W. Howard

Saint Louis University

Peng-Xin Dong

Guangxi Medical University

Yan-Qiu Ou

Guangdong Provincial People's Hospital

Qing-Qing Li

Sun Yat-Sen University

Lu-Yin Wu

Sun Yat-Sen University

Li-Xia Liang

Sun Yat-Sen University

Shuang-Jian Qin

Sun Yat-Sen University

Xiao-Wen Zeng

Sun Yat-Sen University

Li-Wen Hu

Sun Yat-Sen University

Ming-Deng Xiang

Ministry of Environmental Protection of the People's Republic of China

Guang-Hui Dong ( $\sim$ donggh5@mail.sysu.edu.cn ) 


\section{Research Article}

Keywords: 6:2 Cl-PFESA, 8:2 Cl-PFESA, perfluorooctane sulfonate (PFOS) alternatives, overweight/obesity, $\mathrm{BMI}$

Posted Date: October 25th, 2021

DOl: https://doi.org/10.21203/rs.3.rs-977468/v1

License: (c) (i) This work is licensed under a Creative Commons Attribution 4.0 International License. Read Full License 


\section{Abstract \\ Background}

Chlorinated polyfluorinated ether sulfonic acids (Cl-PFESA 6:2 and 8:2), used as perfluorooctane sulfonate (PFOS) alternatives, affect lipid metabolism in vivo and in vitro studies. The association between Cl-PFESAs exposure and the prevalence of overweight /obesity in human is unknown.

\section{Objectives}

We investigated associations of serum 6:2 Cl-PFESA and 8:2 Cl-PFESA with overweight/obesity status in adults.

\section{Methods}

We quantified four perfluoroalkyl substances (PFAS), including 6:2 Cl-PFESA, 8:2 Cl-PFESA, PFOS, and perfluorooctanoic acid (PFOA) in 1275 Chinese adults from the Isomers of C8 Health Project in China study. Participants were categorized into normal weight group [body mass index $(\mathrm{BMI})<25 \mathrm{~kg} / \mathrm{m}^{2}$ ] and overweight/obesity group (BMI $\geq 25 \mathrm{~kg} / \mathrm{m}^{2}$ ).

\section{Results}

Adjusted for potential confounders, BMI in the second quartile of each $\mathrm{In}-\mathrm{ng} / \mathrm{mL}$ greater concentration of 6:2 Cl-PFESA and 8:2 Cl-PFESA were 0.45 [95\% confidence interval (Cl): 0.08, 0.82], and 0.39 (95\% Cl:0.03, $0.76)$ significantly higher than the lowest quartile, respectively. Cl-PFESAs displayed inverted U-shaped associations with the risk of overweight/obesity, and the inflection point of 6:2 Cl-PFESA and 8:2 ClPFESA were $1.80 \mathrm{ng} / \mathrm{mL}, 0.01 \mathrm{ng} / \mathrm{mL}$, respectively. PFOS was associated with waist circumference (WC) but not BMI in each quartile. For PFOA, the associations with outcomes were linearly positive $(P$ for trend $<0.05)$.

\section{Conclusions}

This study reports the first observations on non-monotonic associations between serum 6:2 Cl-PFESA and 8:2 Cl-PFESA concentrations and the prevalence of overweight/obesity in adults. More epidemiological investigations are required to confirm the observed associations.

\section{Introduction}


Obesity is a global public-health problem that increases mortality, decreases life expectancy, and elevates risk for many comorbidities including type 2 diabetes, metabolic syndrome, reproductive abnormalities, hypertension, coronary heart disease, cancers and others (Flegal et al. 2013; Haslam and James 2005; Riaz et al. 2018). The trends in obesity prevalence have been growing over the past 40 years, with $39 \%$ of the global adult population becoming overweight and 13\% becoming obese in 2016 (Jaacks et al. 2019; WHO 2021). The etiology of obesity is intricate. It is not simply an energy imbalance, but is also related to ubiquitous obesogens, such as poly- and perfluoroalkyl substances (PFAS) (Heindel and Blumberg 2019). PFAS are a series of highly fluorinated aliphatic compounds with wide distribution, bio-accumulative properties and extreme environmental persistence (Dhore and Murthy 2021), among which perfluorooctanoic acid (PFOA) and perfluorooctane sulfonate (PFOS) are the most commonly studied and used (Sunderland et al. 2019). Previous literature suggests the association of exposure to PFOS and PFOA with increasing body mass index (BMI) and overweight/obesity (Jain 2014; Tian et al. 2019), though conclusions are not consistent (Chen et al. 2019; Lin et al. 2009; Timmermann et al. 2014).

Chlorinated polyfluorinated ether sulfonic acids (Cl-PFESAs, commercially named F-53B), including 6:2 ClPFESA and 8:2 Cl-PFESA, have been used as a mist suppressant in China for over 40 years as PFOS alternatives. Because of an oxygen atom in the perfluoroalkyl chain, Cl-PFESAs were presupposed less persistent environmentally (Brase et al. 2021; Wang et al. 2013). Due to global actions to phase out PFOS and PFOA, many alternatives are being used in increasing quantities. However, there is increasing evidence that the health risks and toxicity of Cl-PFESAs may be greater than PFOS (Chu et al. 2020; Cui et al. 2018; Shi et al. 2016; Zhang et al. 2018). Yao et al (Yao et al. 2020) reported that exposure to ClPFESAs was significantly positively associated with cholesterol, low-density lipoprotein cholesterol and triglycerides. Evidence in vitro and in vivo indicates that Cl-PFESAs might disrupt lipid metabolism, as $\mathrm{Cl}$ PFESAs affected both metabolic transcription and organismal metabolic phenotype in fertilized zebrafish embryos (Tu et al. 2019), and elevated the relative triglyceride content in mouse 3T3-L1 preadipocyte (Li et al. 2018). However, there are few epidemiological studies currently to assess the risk of adiposity exposure to Cl-PFESAs in human beings.

To address this research gap, we explored the associations between exposure to Cl-PFESAs and overweight status in Isomers of C8 Health Project Study among Chinese adults. We hypothesized that exposure to 6:2 Cl-PFESA and 8:2 Cl-PFESA would be positively associated with BMI, waist circumferences (WC), and risks for being overweight/obese.

\section{Materials And Methods}

\subsection{Study population}

The Isomers of C8 Health Project in China is a cross-sectional study which recruited residents of Shenyang city, Liaoning province from July 2015 to October 2016. We explored the associations between PFAS exposure and health outcomes among people generally exposed to high PFAS concentrations (Yeung et al. 2006). Details about participants enrollment and data collection have been described 
elsewhere (Bao et al. 2017; Zeeshan et al. 2020). Briefly, following the inclusion criteria, of those aged $\geq$ 35 years with $\geq 5$ years residency at their current address, of 1750 participants enrolled, 1275 (72.9\%) completed the questionnaire assessment, anthropometric measurements, and provided blood samples. The local Institutional Review Board of Sun Yat-Sen University Research Ethics Committee approved our study, and study procedures followed the principles of the Helsinki Declaration.

\subsection{Outcome measurements}

Outcomes included standing height (centimeters), weight (kilograms) and WC (centimeters) measured by physicians following the anthropometric measurements method from the Chinese Ministry of Health (CMoH 2013). Standing height was measured to the nearest 0.1 centimeter using a stadiometer with a vertical backboard and a sliding horizontal head piece. Digital weight scales calibrated by calibration weights were used to measure weight to the nearest 0.1 kilogram. Procedures included positioning the measuring tape around a horizontal plane perpendicular to the bilateral midaxillary line at the midpoint between the lowermost of costal margin and the uppermost lateral border of the ilium, then recording the WC measurement values to the nearest 0.1 centimeter at the end of participants' normal expiration. We calculated BMI as weight divided by height $\left(\mathrm{kg} / \mathrm{m}^{2}\right)$. BMI between 25.0 and $29.9 \mathrm{~kg} / \mathrm{m}^{2}$ corresponds to overweight, and BMI of $\geq 30.0 \mathrm{~kg} / \mathrm{m}^{2}$ is obesity for adults, according to the World Health Organization (WHO 2021). Participants were categorized into an overweight/ obesity group (BMI $\geq 25 \mathrm{~kg} / \mathrm{m}^{2}$ ) and normal group $\left(\mathrm{BMl}<25 \mathrm{~kg} / \mathrm{m}^{2}\right)$.

\subsection{Serum PFAS measurement}

Serum PFAS methods were described in detail in a previous study (Chu et al. 2020). In brief, PFAS including novel and legacy in $0.2 \mathrm{~mL}$ serum were extracted by solid phase extraction and detected by an Agilent ultra-performance liquid chromatography (UPLC) 1290 attached to an Agilent 6495B triplequadrupole tandem mass spectrometer (MS/MS) (Agilent Technologies, Palo Alto, CA, USA). We purchased PFAS standards from Wellington Laboratories (Guelph, ON, Canada). Our detailed analytic approach, including methods for quality control, is described in the Supplementary Information. The limit of detection (LOD) of PFAS was set as the minimum detectable concentration in PFAS samples requested to attain a signal-to-noise ratio of $3(\mathrm{~S} / \mathrm{N}=3)$. Table $\mathrm{S} 1$ provides abbreviation and detection parameters of the studied PFAS. PFAS concentrations were replaced with LOD divided by the square root of 2 , when lower than the LOD (Hornung 1990).

\subsection{Statistics}

Distributions were characterized for demographic features, socioeconomic characteristics, behavioral habits, outcomes and exposures. We used Q-Q plots and Shapiro-Wilk tests to evaluate the normality for continuous variables. Due to right-skewed distributions, serum PFAS concentrations were natural logtransformed. To explore associations of BMI, WC and overweight/obesity with PFAS, we used restricted cubic spline (RCS) with three knots at the 10th, 50th, and 90th percentiles to modify models. We found possible non-linear associations between PFAS exposure and outcomes except for PFOA (Figure S1), so we calculated categorical quartiles of PFAS concentrations in models. We conducted generalized additive 
models (GAMs) to estimate correlations of PFAS quartiles as predictors with BMI and WC as outcomes. Logistic regression models were used to estimate odds ratios (OR) and 95\% confidence intervals (Cl) for overweight/obesity as functions of PFAS quartiles. $P$-values for trend were estimated by converting each corresponding PFAS into an ordinal variable in the model. We also used the R "segmented" package to analyze the inflection points for the non-linear associations and then conducted a 2-piecewise binary logistic regression model. We assessed associations between PFAS and outcomes stratified by sex for sensitivity analyses, due to sex-specific effects reported in previous studies (Hales et al. 2017). To evaluate the robustness of our results, we conducted additional analyses by excluding participants for smoking (Audrain-McGovern and Benowitz 2011; Carreras-Torres et al. 2018; Chiolero et al. 2008), alcohol consumption (Sim 2015), and physical inactivity (Basterfield et al. 2014; Hankinson et al. 2010) given changes for BMI, WC and being obese in these groups. Smoking was defined as at least one cigarette per day that lasted for a year. Drinking was defined as $\geq 8 \mathrm{~mL}$ of alcohol (either wine, beer or spirits) per day. Physical activity was defined as self-reported exercise greater than or equal to 60 minutes/day for the past year, or physical inactivity (Tian et al. 2019).

We adjusted essential potential confounding variables in the regression models as factors related to PFAS exposure and outcomes or their precursors. These included age (years, continuous), sex (male/female), education ( $\leq$ high school/> high school), occupation (blue collar/white collar), family income $(<30,000,30,000-100,000$ and $>100,000$ Yuan/year), smoking (yes/no), drinking (yes/no), and physical activity (yes/no). These socio-economic factors and behavioral habits were considered to affect either the sources or the distribution and clearance of PFAS exposure (Brantsaeter et al. 2013; Christensen et al. 2016; Eriksen et al. 2011), that may also affect BMI and WC by changing calorie intake, lipid metabolism, and energy balance (Audrain-McGovern and Benowitz 2011; Carreras-Torres et al. 2018; Chiolero et al. 2008; Sim 2015). Directed acyclic graphs (DAGs) were used to characterize minimally sufficient covariables for reducing confounding bias (Figure S2). The statistical analyses were conducted in R (Version 4.1.0; R Foundation for Statistical Computing, Vienna, Austria) and SAS (version 9.4, SAS Institute Inc., Cary, NC, USA).

\section{Results}

The characteristics of 1275 participants, including demographic characteristics, behavioral habits, outcomes, and selected PFAS concentrations stratified by group, are presented in Table 1. The population was 62 years old on average, principally white-collar workers, high school educated, and $72.6 \%$ from middle-income family (30,0000-100,000 Yuan/year). People in the overweight/obesity group were younger (mean $=59$ years) and more of them were men ( $84.6 \%$ vs. $43.9 \%)$ compared to normal weight group. There was a significantly greater proportion of participants who smoked $(P=0.014)$ and drank alcohol $(P=0.001)$ in the overweight/obesity group. Average BMI was $24.6 \mathrm{~kg} / \mathrm{m}^{2}$, with an average WC of $87.5 \mathrm{~cm}$ overall. Except for 8:2 Cl-PFESA (70.1\%), PFAS in all participants' serum were measured above the LODs (Table S1). The dominant PFAS in serum was PFOS $(24.67 \mathrm{ng} / \mathrm{mL})$. Table S2 lists the Spearman correlation coefficients between PFAS. 
Table 1

Distribution of demographic, lifestyle factors and PFAS concentrations among participants by overweight/obesity

\begin{tabular}{|c|c|c|c|c|}
\hline Characteristic & $\begin{array}{l}\text { Total } \\
(n=1275)\end{array}$ & $\begin{array}{l}\text { Normal } \\
(n=931)\end{array}$ & $\begin{array}{l}\text { Overweight/obesity } \\
d \\
(n=344)\end{array}$ & $P$ \\
\hline Age (year) ${ }^{a}$ & $61.63 \pm 14.64$ & $62.45 \pm 13.92$ & $59.42 \pm 16.27$ & 0.002 \\
\hline Sex ${ }^{b}$ & & & & $\begin{array}{l}< \\
0.001\end{array}$ \\
\hline Male & 700 (54.9\%) & 409 (43.93\%) & 291 (84.59\%) & \\
\hline Female & $575(45.1 \%)$ & $522(56.07 \%)$ & $53(15.41 \%)$ & \\
\hline Education ${ }^{b}$ & & & & 0.042 \\
\hline$\leq$ High school & 404 (31.69\%) & $280(30.08 \%)$ & 124 (36.05\%) & \\
\hline > High school & 871 (68.31\%) & 651 (69.92\%) & $220(63.95 \%)$ & \\
\hline Occupation ${ }^{b}$ & & & & 0.367 \\
\hline White Collar & 1072 (84.08\%) & 788 (84.64\%) & $284(82.56 \%)$ & \\
\hline Blue Collar & 203 (15.92\%) & $143(15.36 \%)$ & $60(17.44 \%)$ & \\
\hline Family income ${ }^{b}$ & & & & 0.407 \\
\hline$<30,000$ & $172(13.49 \%)$ & $129(13.86 \%)$ & $43(12.50 \%)$ & \\
\hline $30,000-100,000$ & 925 (72.55\%) & 679 (72.93\%) & $246(71.51 \%)$ & \\
\hline$>100,000$ & $178(13.96 \%)$ & $123(13.21 \%)$ & 55 (15.99\%) & \\
\hline Smoking (Yes) ${ }^{b}$ & 137 (10.75\%) & $88(9.45 \%)$ & $49(14.24 \%)$ & 0.014 \\
\hline Drinking $\left(\right.$ Yes) ${ }^{b}$ & $321(25.18 \%)$ & $211(22.66 \%)$ & 110 (31.98\%) & 0.001 \\
\hline
\end{tabular}

Abbreviations: PFAS, Perfluoroalkyl and polyfluoroalkyl substance; BMI, body mass index; WC, waist circumference; CI-PFESA, chlorinated polyfluorinated ether sulfonic acids; PFOS, perfluorooctane sulfonate; PFOA, perfluorooctanoic acid.

a Values are mean \pm SD; difference tested using Student's $t$-test.

${ }^{b}$ Values are $\mathrm{n}(\%)$; difference tested using Chi-square test.

${ }^{c}$ Values are median (interquartile range), difference tested using Wilcoxon rank-sum test.

${ }^{\mathrm{d}} \mathrm{BMI} \geq 25 \mathrm{~kg} / \mathrm{m}^{2}$ 


\begin{tabular}{|c|c|c|c|c|}
\hline Characteristic & $\begin{array}{l}\text { Total } \\
(\mathrm{n}=1275)\end{array}$ & $\begin{array}{l}\text { Normal } \\
(n=931)\end{array}$ & $\begin{array}{l}\text { Overweight/obesity } \\
d \\
(n=344)\end{array}$ & $P$ \\
\hline $\begin{array}{l}\text { Physical activity (Yes) } \\
\text { b }\end{array}$ & $404(31.69 \%)$ & $294(31.58 \%)$ & $110(31.98 \%)$ & 0.892 \\
\hline Weight $(\mathrm{kg})^{a}$ & $69.88 \pm 9.70$ & $66.09 \pm 13.92$ & $79.74 \pm 9.83$ & $\begin{array}{l}<.001 \\
0.001\end{array}$ \\
\hline Height $(\mathrm{cm})^{a}$ & $168.64 \pm 6.33$ & $168.21 \pm 5.49$ & $169.77 \pm 8.02$ & 0.001 \\
\hline $\mathrm{BMI}\left(\mathrm{kg} / \mathrm{m}^{2}\right)^{\mathrm{a}}$ & $24.59 \pm 2.61$ & $23.43 \pm 1.63$ & $27.61 \pm 2.23$ & $\grave{0} 001$ \\
\hline$W C(\mathrm{~cm})^{a}$ & $87.52 \pm 7.43$ & $84.67 \pm 5.39$ & $94.89 \pm 6.92$ & $\stackrel{<}{<.001}$ \\
\hline $6: 2$ Cl-PFESA $^{\mathrm{c}}$ & $1.75(1.21,2.50)$ & $1.75(1.13,2.51)$ & $1.75(1.46,2.37)$ & 0.356 \\
\hline 8:2 Cl-PFESA ${ }^{c}$ & $0.01(.001,0.02)$ & $0.01(.001,0.03)$ & $0.01(.001,0.02)$ & 0.256 \\
\hline PFOS $^{c}$ & $\begin{array}{l}24.67(17.38 \\
35.20)\end{array}$ & $\begin{array}{l}24.52(16.78 \\
35.86)\end{array}$ & $25.26(18.14,34.21)$ & 0.164 \\
\hline PFOA ${ }^{c}$ & $4.81(3.58,7.43)$ & $4.72(3.49,6.80)$ & $5.56(3.90,8.91)$ & 0.002 \\
\hline \multicolumn{5}{|c|}{$\begin{array}{l}\text { Abbreviations: PFAS, Perfluoroalkyl and polyfluoroalkyl substance; BMI, body mass index; WC, waist } \\
\text { circumference; Cl-PFESA, chlorinated polyfluorinated ether sulfonic acids; PFOS, perfluorooctane } \\
\text { sulfonate; PFOA, perfluorooctanoic acid. }\end{array}$} \\
\hline \multicolumn{5}{|c|}{ a Values are mean \pm SD; difference tested using Student's $t$-test. } \\
\hline \multicolumn{5}{|c|}{ b Values are $\mathrm{n}(\%)$; difference tested using Chi-square test. } \\
\hline \multicolumn{5}{|c|}{ c Values are median (interquartile range), difference tested using Wilcoxon rank-sum test. } \\
\hline${ }^{d} \mathrm{BMI} \geq 25 \mathrm{~kg} / \mathrm{m}^{2}$ & & & & \\
\hline
\end{tabular}

After adjusting confounders, participants with serum concentrations of 6:2 Cl-PFESA and 8:2 Cl-PFESA in the second quartile had higher BMI compared with those who had serum concentrations in the lowest quartile in models (Table 2). For example, participants' BMI in the second quartile of 6:2 Cl-PFESA were 0.45 (95\% Cl: $0.08,0.82)$, significantly higher than those in the reference group. The continuous trends for associations of Cl-PFESAs exposure with BMI and WC were null ( $P$ for trend $>0.05$ ). PFOS was not associated with BMI but was associated with WC in each quartile. For PFOA, the associations with outcomes were linear $(P$ for trend $<0.05$ ). Table 2 shows the adjusted ORs with $95 \% \mathrm{Cls}$ of overweight/obesity with categorical serum PFAS concentrations. We detected 1.80 -fold ( $95 \% \mathrm{Cl}: 1.23$, 2.63) greater odds of overweight/obesity in the second quartile of $6: 2 \mathrm{Cl}$-PFESA compared to the reference group, which had not been found significant in the higher quartile. 
Table 2

Associations of BMI $\left(\mathrm{kg} / \mathrm{m}^{2}\right)$, WC $(\mathrm{cm})$ and overweight or obesity $\left(\mathrm{BMI} \geq 25 \mathrm{~kg} / \mathrm{m}^{2}\right.$ ) with serum PFAS (In $\mathrm{ng} / \mathrm{mL})(\mathrm{n}=1275)$

\begin{tabular}{|c|c|c|c|}
\hline \multirow{2}{*}{$\begin{array}{l}\text { PFAS } \\
\text { Quartile (ln ng/mL) }\end{array}$} & BMI $\left(\mathrm{kg} / \mathrm{m}^{2}\right)$ & WC (cm) & Overweight/obesity \\
\hline & $\beta(95 \% \mathrm{Cl})^{a}$ & $\beta(95 \% \mathrm{Cl})^{a}$ & Adjusted OR $(95 \% \mathrm{Cl})^{a}$ \\
\hline \multicolumn{4}{|l|}{ 6:2 Cl-PFESA } \\
\hline$\leq 1.21$ & Reference & Reference & 1.00 (reference) \\
\hline$>1.21$ to 1.75 & $0.47(0.09,0.84)$ & $1.62(0.58,2.65)$ & $1.80(1.23,2.63)$ \\
\hline$>1.75$ to 2.50 & $0.40(-0.04,0.84)$ & $1.03(-0.20,2.26)$ & $1.41(0.89,2.21)$ \\
\hline$>2.50$ & $0.29(-0.11,0.69)$ & $1.24(0.13,2.35)$ & $1.21(0.81,1.82)$ \\
\hline$P$ for trend ${ }^{b}$ & 0.274 & 0.109 & 0.276 \\
\hline \multicolumn{4}{|l|}{ 8:2 Cl-PFESA } \\
\hline$\leq 0.001$ & Reference & Reference & 1.00 (reference) \\
\hline$>0.001$ to 0.01 & $0.39(0.03,0.76)$ & $0.93(-0.09,1.95)$ & $1.39(0.98,1.96)$ \\
\hline$>0.01$ to 0.02 & $0.02(-0.41,0.45)$ & $0.11(-1.09,1.30)$ & $0.78(0.50,1.21)$ \\
\hline$>0.02$ & $0.17(-0.21,0.54)$ & $0.45(-0.60,1.50)$ & $0.96(0.66,1.39)$ \\
\hline$P$ for trend ${ }^{b}$ & 0.705 & 0.661 & 0.350 \\
\hline \multicolumn{4}{|l|}{ PFOS } \\
\hline$\leq 17.38$ & Reference & Reference & 1.00 (reference) \\
\hline$>17.38$ to 24.67 & $0.29(-0.11,0.68)$ & $1.72(0.63,2.82)$ & $1.05(0.71,1.56)$ \\
\hline$>24.67$ to 35.20 & $0.34(-0.06,0.74)$ & $1.84(0.74,2.95)$ & $1.02(0.69,1.50)$ \\
\hline$>35.20$ & $0.04(-0.36,0.44)$ & $1.67(0.56,2.78)$ & $0.86(0.57,1.28)$ \\
\hline$P$ for trend ${ }^{b}$ & 0.809 & 0.005 & 0.810 \\
\hline \multicolumn{4}{|l|}{ PFOA } \\
\hline$\leq 3.58$ & Reference & Reference & 1.00 (reference) \\
\hline
\end{tabular}

Abbreviations: PFAS, Perfluoroalkyl and polyfluoroalkyl substance; BMI, body mass index; WC, waist circumference; CI-PFESA, chlorinated polyfluorinated ether sulfonic acids; PFOS, perfluorooctane sulfonate; PFOA, perfluorooctanoic acid; OR, odds ratios; $\mathrm{Cl}$, confidence intervals.

a Adjusted for sex, age, education, occupation, family income, smoking, drinking, and physical activity.

b Tested using the median value for each category. 


\begin{tabular}{|c|c|c|c|}
\hline \multirow{2}{*}{$\begin{array}{l}\text { PFAS } \\
\text { Quartile (ln ng/mL) }\end{array}$} & $\mathrm{BMI}\left(\mathrm{kg} / \mathrm{m}^{2}\right)$ & WC (cm) & Overweight/obesity \\
\hline & $\beta(95 \% \mathrm{Cl})^{a}$ & $\beta(95 \% \mathrm{Cl})^{a}$ & Adjusted OR (95\%Cl) a \\
\hline$>3.58$ to 4.81 & $0.08(-0.31,0.48)$ & $0.32(-0.76,1.40)$ & $1.06(0.72,1.58)$ \\
\hline$>4.81$ to 7.43 & $0.21(-0.18,0.61)$ & $1.18(0.09,2.27)$ & $1.00(0.67,1.49)$ \\
\hline$>7.43$ & $0.61(0.21,1.01)$ & $2.19(1.07,3.30)$ & $1.68(1.14,2.49)$ \\
\hline$P$ for trend ${ }^{b}$ & 0.003 & $<0.001$ & 0.003 \\
\hline \multicolumn{4}{|c|}{$\begin{array}{l}\text { Abbreviations: PFAS, Perfluoroalkyl and polyfluoroalkyl substance; BMI, body mass index; WC, waist } \\
\text { circumference; Cl-PFESA, chlorinated polyfluorinated ether sulfonic acids; PFOS, perfluorooctane } \\
\text { sulfonate; PFOA, perfluorooctanoic acid; OR, odds ratios; Cl, confidence intervals. }\end{array}$} \\
\hline \multicolumn{4}{|c|}{ a Adjusted for sex, age, education, occupation, family income, smoking, drinking, and physical activity. } \\
\hline
\end{tabular}

Figure $1 \mathrm{~A}$ and $\mathrm{B}$ also described the non-monotonic relationship and the inflection points of $6: 2 \mathrm{Cl}$-PFESA and 8:2 Cl-PFESA exposure with overweight/obesity, respectively. The risk of overweight/obesity increased $(\mathrm{OR}=1.94 ; 95 \% \mathrm{Cl}: 1.24,3.01)$ until around $1.80 \mathrm{ng} / \mathrm{mL}$ of predicted $6: 2 \mathrm{Cl}$-PFESA concentration and then decreased $(\mathrm{OR}=0.69 ; 95 \% \mathrm{Cl}$ : $0.39,1.21)$. Similarly, below $0.01 \mathrm{ng} / \mathrm{mL}$ of $8: 2 \mathrm{Cl}$-PFESA exposure, the OR of overweight/obesity was $1.24(95 \% \mathrm{Cl}: 1.05,1.46)$. The relationship between Cl-PFESAs exposure and overweight/ obesity was strongly inverted U-shaped.

The results of crude regression models were generally consistent with the main analysis (Table S3). There were slightly different associations between PFAS and overweight/obesity status stratified by sex (Table S4-5). When the participants who smoked and drank were excluded, results were similar (Table S67), but the associations among PFAS and outcomes in the subgroup of physical activity were weaker than in the main analysis (Table S8).

\section{Discussion}

In this cross-sectional study, our results indicated that Cl-PFESAs exposure was associated with overweight/obesity status. We found that serum concentrations of 6:2 Cl-PFESA and 8:2 Cl-PFESA in the second quartile had higher BMI compared with these in the lowest quartile, but not in the higher quartiles, suggesting the non-monotonic relationship between exposure to Cl-PFESAs and overweight/obesity incidence. Cl-PFESAs displayed an inverse U-shape association with the prevalence of overweight/obesity. Our study also demonstrated that exposure to PFOA was linearly positively associated with each outcome, and PFOS was only positively associated waist circumference. This is the first study to report the non-monotonic associations between Cl-PFESAs exposure and overweight status in human populations. 
Among 1275 Chinese adults' serum, 6:2 Cl-PFESA, PFOA, and PFOS were all detected and 8:2 Cl-PFESA was detected in $70 \%$ of participants. A recent review summarized Cl-PFESAs concentrations in China, the means of which are $4.20,102,941 \mathrm{ng} / \mathrm{mL}$ in the general population, high fish consumers, and metal plating workers, respectively (Brase et al. 2021). In Shandong province of China, the 6:2 Cl-PFESA level of 977 residents living near a fluorochemical plant was $2.311 \mathrm{ng} / \mathrm{mL}$ (Yao et al. 2020). These were slightly higher than our results. But the median value of $6: 2$ Cl-PFESA concentrations was $0.34 \mathrm{ng} / \mathrm{mL}$ that lower than ours among 519 pregnant women in Shanxi, China (X Liu et al. 2020). The concentrations of ClPFESAs varied regionally (Chen et al. 2017; Chu et al. 2020; X Liu et al. 2020; Pan et al. 2017). The serum PFOA and PFOS concentrations found by the current study were higher than those reported in most other national studies (Schulz et al. 2020).

To date, there are no human studies available to explore the association between the levels of CI-PEFSA and overweight/obesity status, but several studies have reported the associations of exposure to PFOS and PFOA with obesity outcomes (Averina et al. 2021; Chen et al. 2019; Christensen et al. 2016; Eriksen et al. 2011; Geiger et al. 2021; Jain 2014; Lin et al. 2009; Liu et al. 2018; Nelson et al. 2010; Timmermann et al. 2014). We summarize them in Table S7. In line with our results, Geiger et al (Geiger et al. 2021) reported greater PFOA exposure was associated with higher risk of overweight/obesity in US children during 1999-2012 ( $\left.\mathrm{OR}_{\mathrm{Q} 3 \text { vs. } \mathrm{Q} 1}=2.22,95 \% \mathrm{Cl}: 1.20,4.13 ; \mathrm{OR}_{\mathrm{Q} 4 \text { vs. } \mathrm{Q} 1}=2.73,95 \% \mathrm{Cl}: 1.10,6.74\right)$. Also consistent with our studies, increased PFOA levels measured in 5591 US people aged 12 and older was associated with increases in BMI $(P=0.038)$ (Jain 2014). However, the relationship of human exposure and overweight/ obesity status is still controversial, given concerns about reverse causality and effective dose (Jain 2020). Due to different modeling approaches and inconsistent confounding variables, the results of some studies were not consistent with ours (Averina et al. 2021; Chen et al. 2019; Christensen et al. 2016; Eriksen et al. 2011; Lin et al. 2009; Timmermann et al. 2014). For example, a cross-sectional study about the associations between PFAS concentrations in Norwegian adolescents and obesity, without adjustment for socioeconomic status, showed null associations for PFOS and PFOA (Averina et al. 2021).

Though there is no evidence that showed Cl-PFESAs might be potential obesogens in human beings, our previous epidemiologic studies found serum CI-PFESAs concentrations were significantly positively associated with serum lipids (Cong et al. 2021) and the risk of metabolic syndrome (MetS) (Yu et al. 2021). In addition, Yao et al (Yao et al. 2020) reported multivariate linear regression coefficients of $9.80 \%$ (95\% Cl: $6.09,13.63)$ for cholesterol ( $\mathrm{mmol} / \mathrm{L}), 9.59 \%$ (95\% Cl: 5.29, 14.07) for low-density lipoprotein cholesterol (mmol/L), $12.34 \%$ (95\% Cl: $2.80,2.88)$ for triglycerides (mmol/L), higher 6:2 Cl-PFESA per In$\mathrm{ng} / \mathrm{mL}$ concentrations, respectively, which suggests potential elevated lipid parameters from exposure to 6:2 Cl-PFESA. Furthermore, F-53B has a stronger metabolism-disrupting effect than PFOS in fertilized zebrafish embryos, affecting both metabolic transcription and organismal metabolic phenotype (Tu et al. 2019). A study in vitro indicated that Cl-PFESAs elevated the relative triglyceride content in mouse 3T3-L1 preadipocyte with adipogenesis promotion potency greater than PFOS (Li et al. 2018). 
Potential biological effects were described in previous studies that could reveal the hazard of Cl-PFESAs exposure, although the mechanisms promoting adiposity risk by Cl-PFESAs are not clear. 6:2 Cl-PFESA displayed toxic effects on human liver $\mathrm{HL}-7702$ cell, and significantly up-regulated gene $\mathrm{Cd} 36$ expression regulated long-chain fatty acids transportation through the adipocyte plasma membrane(Sheng et al. 2018). Cl-PFESAs also affected osteogenic differentiation in human bone mesenchymal stem cells (hBMSCs) related to obesity and metabolic diseases (Pan et al. 2019). In several in vivo and in vitro investigations, Cl-PFESAs showed agonistic activity toward the peroxisome proliferator-activated receptors (PPARs) pathways related lipid metabolism (Li et al. 2018; Sheng et al. 2018; Shi et al. 2019). Cl-PFESAs also have the characteristics of endocrine disruptors, which might be associated with glucocorticoids and progestogens synthesis in neonates (H Liu et al. 2020), sex hormone disorders in adult men (Cui et al. 2020), and induced estrogenic effects in zebrafish (Xin et al. 2020). The endocrine system is important for energy balance, fat distribution and fat deposition. For example, sex hormones affect food intake and alter the balance of glucose and insulin, lipogenesis and lipolysis to cause obesity (Heindel and Blumberg 2019). Endocrine disrupting chemicals (EDCs) are considered as obesogens promoting obesity in humans or animals (Nadal et al. 2017). However, we need more data before identifying $\mathrm{Cl}$-PFESAs as an obesogen.

Interestingly, Cl-PFESAs displayed an inverted U-shaped relationship with the prevalence of overweight/obesity in our study. This unconventional dose-response relationship called non-monotonic dose-response (NMDR) relationship is normal in studies investigating the effects of EDCs (Lagarde et al. 2015; Vassilopoulou et al. 2017). Although there is less data about NMDR of Cl-PFESAs, several studies had reported that NMDR relationships occurred between PFAS and health outcomes. For example, Liao et al found a J-shaped relationship of PFOA and PFNA with the risk of hypertension among adults in US (Liao et al. 2020). PFOS and progesterone levels displayed an inverse U-shape dose-response relationship in neonates (H Liu et al. 2020). NMDR relationship indicates the possibility of Cl-PFESAs with endocrine disrupting characteristics, and this exposure-outcome association is a challenge for risk assessment of Cl-PFESAs.

Our results were based on a community-based cross-sectional study with a relatively large population, which could reduce the occurrence of random errors. Moreover, we enrolled a comprehensive panel of potential confounders, consistent with previous studies, exploring the association between exposure to PFAS and overweight/obesity, including sociodemographic and behavior factors. Finally, we accounted for non-monotonic association of PFAS with the prevalence of overweight/obesity using a restricted cubic spline regression analysis and 2-piecewise binary logistic regression for statistical analyses.

However, several limitations should not be ignored in this study. First, there may be reverse causality in our cross-sectional study design. However, it is difficult for PFAS to accumulate in adipose tissue with lipophobic properties (Aas et al. 2014; Fabrega et al. 2014; Wu et al. 2019). Lipid mobilization and not fat could effect PFAS concentrations in blood and other tissues (Aas et al. 2014). Second, although we included key confounding variables in the models, we did not adjust for diets, which could change PFAS exposure due to different PFAS-containing food consumption, dietary quality, and energy intake 
(Christensen et al. 2016; Eriksen et al. 2011). Third, we did not consider the cocktail effect of various pollutants that may affect overweight/obesity, such as polychlorinated biphenyls (PCBs), phthalates and other POPs, as EDCs, which might act in a synergistic or antagonistic manner to impact PFAS metabolic disorder effects (Chamorro-Garcia and Veiga-Lopez 2021; Choi et al. 2021; Egusquiza and Blumberg 2020). Bayesian kernel machine regression (BKMR) models performed to analyze multiple-chemical exposures will be necessary for further research in a larger population. Fourth, we conducted a common but controversial approach that imputed values below detection limits by LOD/ $\sqrt{2}$ (Huynh et al. 2014; Richardson and Ciampi 2003; Schisterman et al. 2006), due to the detection proportion of 8:2 Cl-PFESA being $70.12 \%$. However, our results were similar by using multiple imputing values below LOD for a repeat analysis (Table S10), although modestly stronger for the second quartile of 8:2 Cl-PFESA. Fifth, the population in this study was in China, hence future research conducted in other areas is needed.

\section{Conclusion}

Our study suggested that exposure to Cl-PFESAs displayed an inverted U-shaped association with the prevalence of overweight/obesity in Chinese adults. However, our results were based on a cross-sectional study with participants from only one city. Larger studies are needed to identify metabolic disorder effects and the active doses of Cl-PFESAs in the future. The alternatives to PFOS are not anticipated to be less toxic than PFOS, so the widespread use of Cl-PFESAs is a growing concern.

\section{Declarations}

\section{Acknowledgments:}

The authors thank all of participants in this study. The research was funded by the National Natural Science Foundation of China (No.82173471; No.82003409; No.82073503; No. M-0420; No.81903287; No. 81950410633), Fundamental Research Funds for the Central Universities (19ykjc01), Natural Science Foundation of Guangdong Province (No. 2021A1515012212; No. 2021A151011754; No. 2021B1515020015; No. 2020A1515011131; No. 2019A050510017; No. 2018B05052007; No. 2017A090905042), the Science and Technology Program of Guangzhou (No. 201807010032; No. 201803010054; No. 201903010023).

\section{Statements and Declarations:}

The authors declare that they have no known competing financial interests or personal relationships that could have appeared to influence the work reported in this paper.

\section{Data Availability Statement!}

The raw data supporting the conclusions will be made available by the corresponding author (Guang-Hui Dong) on reasonable request. 


\section{References}

1. Aas CB, Fuglei E, Herzke D, Yoccoz NG, Routti H (2014) Effect of body condition on tissue distribution of perfluoroalkyl substances (pfass) in arctic fox (vulpes lagopus). Environ Sci Technol 48:1165411661. http://dx.doi.org/10.1021/es503147n

2. Audrain-McGovern J, Benowitz NL (2011) Cigarette smoking, nicotine, and body weight. Clin Pharmacol Ther 90:164-168. http://dx.doi.org/10.1038/clpt.2011.105

3. Averina M, Brox J, Huber S, Furberg AS (2021) Exposure to perfluoroalkyl substances (pfas) and dyslipidemia, hypertension and obesity in adolescents. The fit futures study. Environ Res 195:110740. http://dx.doi.org/10.1016/j.envres.2021.110740

4. Bao WW, Qian ZM, Geiger SD, Liu E, Liu Y, Wang SQ et al (2017) Gender-specific associations between serum isomers of perfluoroalkyl substances and blood pressure among chinese: Isomers of c8 health project in china. Sci Total Environ 607-608:1304-1312.

http://dx.doi.org/10.1016/j.scitotenv.2017.07.124

5. Basterfield L, Jones AR, Parkinson KN, Reilly J, Pearce MS, Reilly JJ et al (2014) Physical activity, diet and bmi in children aged 6-8 years: A cross-sectional analysis. BMJ Open 4:e005001. http://dx.doi.org/10.1136/bmjopen-2014-005001

6. Brantsaeter AL, Whitworth KW, Ydersbond TA, Haug LS, Haugen M, Knutsen HK et al (2013) Determinants of plasma concentrations of perfluoroalkyl substances in pregnant norwegian women. Environ Int 54:74-84. http://dx.doi.org/10.1016/j.envint.2012.12.014

7. Brase RA, Mullin EJ, Spink DC (2021) Legacy and emerging per- and polyfluoroalkyl substances: Analytical techniques, environmental fate, and health effects. Int J Mol Sci 22:995. http://dx.doi.org/10.3390/ijms22030995

8. Carreras-Torres R, Johansson M, Haycock PC, Relton CL, Davey Smith G, Brennan P et al (2018) Role of obesity in smoking behaviour: Mendelian randomisation study in uk biobank. Bmj:k1767. http://dx.doi.org/10.1136/bmj.k1767

9. Chamorro-Garcia R, Veiga-Lopez A (2021) The new kids on the block: Emerging obesogens. Adv Pharmacol 92:457-484. http://dx.doi.org/10.1016/bs.apha.2021.05.003

10. Chen A, Jandarov R, Zhou L, Calafat AM, Zhang G, Urbina EM et al (2019) Association of perfluoroalkyl substances exposure with cardiometabolic traits in an island population of the eastern adriatic coast of croatia. Sci Total Environ 683:29-36. http://dx.doi.org/10.1016/j.scitotenv.2019.05.250

11. Chen F, Yin S, Kelly BC, Liu W (2017) Chlorinated polyfluoroalkyl ether sulfonic acids in matched maternal, cord, and placenta samples: A study of transplacental transfer. Environ Sci Technol 51:6387-6394. http://dx.doi.org/10.1021/acs.est.6b06049

12. Chiolero A, Faeh D, Paccaud F, Cornuz J (2008) Consequences of smoking for body weight, body fat distribution, and insulin resistance. Am J Clin Nutr 87:801-809.

http://dx.doi.org/10.1093/ajcn/87.4.801

Page $14 / 21$ 
13. Choi SI, Kwon HY, Han X, Men X, Choi YE, Jang GW et al (2021) Environmental obesogens (bisphenols, phthalates and parabens) and their impacts on adipogenic transcription factors in the absence of dexamethasone in 3t3-I1 cells. J Steroid Biochem Mol Biol 214:105994. http://dx.doi.org/10.1016/j.jsbmb.2021.105994

14. Christensen KY, Raymond M, Thompson BA, Anderson HA (2016) Perfluoroalkyl substances in older male anglers in wisconsin. Environ Int 91:312-318. http://dx.doi.org/10.1016/j.envint.2016.03.012

15. Chu C, Zhou Y, Li Q-Q, Bloom MS, Lin S, Yu Y-J et al (2020) Are perfluorooctane sulfonate alternatives safer? New insights from a birth cohort study. Environ Int 135:105365. http://dx.doi.org/10.1016/j.envint.2019.105365

16. $\mathrm{CMoH}$ (2013) Chinese ministry of health. Anthropometric measurements method in health surveillance.

17. Cong J, Chu C, Li QQ, Zhou Y, Min Qian Z, Dee Geiger S et al (2021) Associations of perfluorooctane sulfonate alternatives and serum lipids in chinese adults. Environ Int 155:106596. http://dx.doi.org/10.1016/j.envint.2021.106596

18. Cui Q, Pan Y, Zhang H, Sheng N, Wang J, Guo Y et al (2018) Occurrence and tissue distribution of novel perfluoroether carboxylic and sulfonic acids and legacy per/polyfluoroalkyl substances in black-spotted frog (pelophylax nigromaculatus). Environ Sci Technol 52:982-990. http://dx.doi.org/10.1021/acs.est.7b03662

19. Cui Q, Pan Y, Wang J, Liu H, Yao B, Dai J (2020) Exposure to per- and polyfluoroalkyl substances (pfass) in serum versus semen and their association with male reproductive hormones. Environ Pollut 266:115330. http://dx.doi.org/10.1016/j.envpol.2020.115330

20. Dhore R, Murthy GS (2021) Per/polyfluoroalkyl substances production, applications and environmental impacts. Bioresour Technol 341:125808.

http://dx.doi.org/10.1016/j.biortech.2021.125808

21. Egusquiza RJ, Blumberg B (2020) Environmental obesogens and their impact on susceptibility to obesity: New mechanisms and chemicals. Endocrinology 161:bqaa024. http://dx.doi.org/10.1210/endocr/bqaa024

22. Eriksen KT, Sorensen M, McLaughlin JK, Tjonneland A, Overvad K, Raaschou-Nielsen $O$ (2011) Determinants of plasma pfoa and pfos levels among 652 danish men. Environ Sci Technol 45:8137-8143. http://dx.doi.org/10.1021/es100626h

23. Fabrega F, Kumar V, Schuhmacher M, Domingo JL, Nadal M (2014) Pbpk modeling for pfos and pfoa: Validation with human experimental data. Toxicol Lett 230:244-251. http://dx.doi.org/10.1016/j.toxlet.2014.01.007

24. Flegal KM, Kit BK, Orpana H, Graubard BI (2013) Association of all-cause mortality with overweight and obesity using standard body mass index categories a systematic review and meta-analysis. Jama-J Am Med Assoc 309:71-82. http://dx.doi.org/10.1001/jama.2012.113905

25. Geiger SD, Yao P, Vaughn MG, Qian Z (2021) Pfas exposure and overweight/obesity among children in a nationally representative sample. Chemosphere 268:128852. 
http://dx.doi.org/10.1016/j.chemosphere.2020.128852

26. Hales CM, Carroll MD, Fryar CD, Ogden CL (2017) Prevalence of obesity among adults and youth: United states, 2015-2016. 1941-4935

27. Hankinson AL, Daviglus ML, Bouchard C, Carnethon M, Lewis CE, Schreiner PJ et al (2010) Maintaining a high physical activity level over 20 years and weight gain. Jama 304:2603-2610. http://dx.doi.org/10.1001/jama.2010.1843

28. Haslam DW, James WPT (2005) Obesity The Lancet 366:1197-1209. http://dx.doi.org/10.1016/s0140-6736(05)67483-1

29. Heindel JJ, Blumberg B (2019) Environmental obesogens: Mechanisms and controversies. Annu Rev Pharmacol Toxicol 59:89-106. http://dx.doi.org/10.1146/annurev-pharmtox-010818-021304

30. Hornung RW, Reed, Laurence D (1990) Estimation of average concentration in the presence of nondetectable values. Appl Occup Environ Hyg 5:46-51.

http://dx.doi.org/10.1080/1047322X.1990.10389587

31. Huynh T, Ramachandran G, Banerjee S, Monteiro J, Stenzel M, Sandler DP et al (2014) Comparison of methods for analyzing left-censored occupational exposure data. Ann Occup Hyg 58:1126-1142. http://dx.doi.org/10.1093/annhyg/meu067

32. Jaacks LM, Vandevijvere S, Pan A, McGowan CJ, Wallace C, Imamura F et al (2019) The obesity transition: Stages of the global epidemic. The Lancet Diabetes Endocrinology 7:231-240. http://dx.doi.org/10.1016/s2213-8587(19)30026-9

33. Jain RB (2014) Contribution of diet and other factors to the levels of selected polyfluorinated compounds: Data from nhanes 2003-2008. Int J Hyg Environ Health 217:52-61 http://dx.doi.org/10.1016/j.ijheh.2013.03.008

34. Jain RB (2020) Impact of the co-occurrence of obesity with diabetes, anemia, hypertension, and albuminuria on concentrations of selected perfluoroalkyl acids. Environ Pollut 266:115207. http://dx.doi.org/10.1016/j.envpol.2020.115207

35. Lagarde F, Beausoleil C, Belcher SM, Belzunces LP, Emond C, Guerbet M et al (2015) Non-monotonic dose-response relationships and endocrine disruptors: A qualitative method of assessment. Environ Health 14:13. http://dx.doi.org/10.1186/1476-069X-14-13

36. Li CH, Ren XM, Ruan T, Cao LY, Xin Y, Guo LH et al (2018) Chlorinated polyfluorinated ether sulfonates exhibit higher activity toward peroxisome proliferator-activated receptors signaling pathways than perfluorooctanesulfonate. Environ Sci Technol 52:3232-3239.

http://dx.doi.org/10.1021/acs.est.7b06327

37. Liao S, Yao W, Cheang I, Tang X, Yin T, Lu X et al (2020) Association between perfluoroalkyl acids and the prevalence of hypertension among us adults. Ecotoxicol Environ Saf 196:110589. http://dx.doi.org/10.1016/j.ecoenv.2020.110589

38. Lin CY, Chen PC, Lin YC, Lin LY (2009) Association among serum perfluoroalkyl chemicals, glucose homeostasis, and metabolic syndrome in adolescents and adults. Diabetes Care 32:702-707. http://dx.doi.org/10.2337/dc08-1816 
39. Liu G, Dhana K, Furtado JD, Rood J, Zong G, Liang L et al (2018) Perfluoroalkyl substances and changes in body weight and resting metabolic rate in response to weight-loss diets: A prospective study. PLoS Med 15:e1002502. http://dx.doi.org/10.1371/journal.pmed.1002502

40. Liu H, Pan Y, Jin S, Li Y, Zhao L, Sun X et al (2020) Associations of per-/polyfluoroalkyl substances with glucocorticoids and progestogens in newborns. Environment International 140. http://dx.doi.org/10.1016/j.envint.2020.105636

41. Liu X, Chen D, Wang B, Xu F, Pang Y, Zhang L et al (2020) Does low maternal exposure to per- and polyfluoroalkyl substances elevate the risk of spontaneous preterm birth? A nested case-control study in china. Environ Sci Technol 54:8259-8268. http://dx.doi.org/10.1021/acs.est.0c01930

42. Nadal A, Quesada I, Tuduri E, Nogueiras R, Alonso-Magdalena P (2017) Endocrine-disrupting chemicals and the regulation of energy balance. Nat Rev Endocrinol 13:536-546. http://dx.doi.org/10.1038/nrendo.2017.51

43. Nelson JW, Hatch EE, Webster TF (2010) Exposure to polyfluoroalkyl chemicals and cholesterol, body weight, and insulin resistance in the general u.S. Population. Environ Health Perspect 118:197-202. http://dx.doi.org/10.1289/ehp.0901165

44. Pan Y, Zhu Y, Zheng T, Cui Q, Buka SL, Zhang B et al (2017) Novel chlorinated polyfluorinated ether sulfonates and legacy per-/polyfluoroalkyl substances: Placental transfer and relationship with serum albumin and glomerular filtration rate. Environ Sci Technol 51:634-644. http://dx.doi.org/10.1021/acs.est.6b04590

45. Pan Y, Qin H, Liu W, Zhang Q, Zheng L, Zhou C et al (2019) Effects of chlorinated polyfluoroalkyl ether sulfonate in comparison with perfluoroalkyl acids on gene profiles and stemness in human mesenchymal stem cells. Chemosphere 237:124402.

http://dx.doi.org/10.1016/j.chemosphere.2019.124402

46. Riaz H, Khan MS, Siddiqi TJ, Usman MS, Shah N, Goyal A et al (2018) Association between obesity and cardiovascular outcomes: A systematic review and meta-analysis of mendelian randomization studies. JAMA Netw Open 1:e183788. http://dx.doi.org/10.1001/jamanetworkopen.2018.3788

47. Richardson DB, Ciampi A (2003) Effects of exposure measurement error when an exposure variable is constrained by a lower limit. Am J Epidemiol 157:355-363. http://dx.doi.org/10.1093/aje/kwf217

48. Schisterman EF, Vexler A, Whitcomb BW, Liu A (2006) The limitations due to exposure detection limits for regression models. Am J Epidemiol 163:374-383. http://dx.doi.org/10.1093/aje/kwj039

49. Schulz K, Silva MR, Klaper R (2020) Distribution and effects of branched versus linear isomers of pfoa, pfos, and pfhxs: A review of recent literature. Sci Total Environ 733:139186. http://dx.doi.org/10.1016/j.scitotenv.2020.139186

50. Sheng N, Cui R, Wang J, Guo Y, Wang J, Dai J (2018) Cytotoxicity of novel fluorinated alternatives to long-chain perfluoroalkyl substances to human liver cell line and their binding capacity to human liver fatty acid binding protein. Arch Toxicol 92:359-369. http://dx.doi.org/10.1007/s00204-0172055-1 
51. Shi G, Cui Q, Wang J, Guo H, Pan Y, Sheng N et al (2019) Chronic exposure to 6:2 chlorinated polyfluorinated ether sulfonate acid (f-53b) induced hepatotoxic effects in adult zebrafish and disrupted the ppar signaling pathway in their offspring. Environ Pollut 249:550-559. http://dx.doi.org/10.1016/j.envpol.2019.03.032

52. Shi Y, Vestergren R, Xu L, Zhou Z, Li C, Liang Y et al (2016) Human exposure and elimination kinetics of chlorinated polyfluoroalkyl ether sulfonic acids (cl-pfesas). Environ Sci Technol 50:2396-2404. http://dx.doi.org/10.1021/acs.est.5b05849

53. Sim F (2015) Alcoholic drinks contribute to obesity and should come with mandatory calorie counts. BMJ 350:h2047 http://dx.doi.org/10.1136/bmj.h2047

54. Sunderland EM, Hu XC, Dassuncao C, Tokranov AK, Wagner CC, Allen JG (2019) A review of the pathways of human exposure to poly- and perfluoroalkyl substances (pfass) and present understanding of health effects. J Expo Sci Environ Epidemiol 29:131-147. http://dx.doi.org/10.1038/s41370-018-0094-1

55. Tian YP, Zeng XW, Bloom MS, Lin S, Wang SQ, Yim SHL et al (2019) Isomers of perfluoroalkyl substances and overweight status among chinese by sex status: Isomers of $c 8$ health project in china. Environ Int 124:130-138. http://dx.doi.org/10.1016/j.envint.2019.01.006

56. Timmermann CA, Rossing LI, Grontved A, Ried-Larsen M, Dalgard C, Andersen LB et al (2014) Adiposity and glycemic control in children exposed to perfluorinated compounds. J Clin Endocrinol Metab 99:E608-E614. http://dx.doi.org/10.1210/jc.2013-3460

57. Tu W, Martinez R, Navarro-Martin L, Kostyniuk DJ, Hum C, Huang J et al (2019) Bioconcentration and metabolic effects of emerging pfos alternatives in developing zebrafish. Environ Sci Technol 53:13427-13439. http://dx.doi.org/10.1021/acs.est.9b03820

58. Vassilopoulou L, Psycharakis C, Petrakis D, Tsiaoussis J, Tsatsakis AM (2017) Obesity, persistent organic pollutants and related health problems. In: Obesity and lipotoxicity, (Engin AB, Engin A, eds). Cham:Springer International Publishing, 81-110

59. Wang S, Huang J, Yang Y, Hui Y, Ge Y, Larssen T et al (2013) First report of a chinese pfos alternative overlooked for 30 years: Its toxicity, persistence, and presence in the environment. Environ Sci Technol 47:10163-10170. http://dx.doi.org/10.1021/es401525n

60. WHO (2021) Obesity and overweight. Available: https://www.who.int/news-room/factsheets/detail/obesity-and-overweight [accessed June 9 2021]

61. Wu Y, Deng M, Jin Y, Liu X, Mai Z, You H et al (2019) Toxicokinetics and toxic effects of a chinese pfos alternative f-53b in adult zebrafish. Ecotoxicol Environ Saf 171:460-466. http://dx.doi.org/10.1016/j.ecoenv.2019.01.010

62. Xin Y, Wan B, Yu B, Fan Y, Chen, Guo LH (2020) Chlorinated polyfluoroalkylether sulfonic acids exhibit stronger estrogenic effects than perfluorooctane sulfonate by activating nuclear estrogen receptor pathways. Environ Sci Technol 54:3455-3464. http://dx.doi.org/10.1021/acs.est.9b07708

63. Yao J, Pan Y, Sheng N, Su Z, Guo Y, Wang J et al (2020) Novel perfluoroalkyl ether carboxylic acids (pfecas) and sulfonic acids (pfesas): Occurrence and association with serum biochemical 
parameters in residents living near a fluorochemical plant in china. Environ Sci Technol 54:1338913398. http://dx.doi.org/10.1021/acs.est.0c02888

64. Yeung LW, So MK, Jiang G, Taniyasu S, Yamashita N, Song M et al (2006) Perfluorooctanesulfonate and related fluorochemicals in human blood samples from china. Environ Sci Technol 40:715-720. http://dx.doi.org/10.1021/es052067y

65. Yu S, Feng WR, Liang ZM, Zeng XY, Bloom MS, Hu GC et al (2021) Perfluorooctane sulfonate alternatives and metabolic syndrome in adults: New evidence from the isomers of $c 8$ health project in china. Environ Pollut 283:117078. http://dx.doi.org/10.1016/j.envpol.2021.117078

66. Zeeshan M, Yang Y, Zhou Y, Huang W, Wang Z, Zeng XY et al (2020) Incidence of ocular conditions associated with perfluoroalkyl substances exposure: Isomers of $c 8$ health project in china. Environ Int 137:105555. http://dx.doi.org/10.1016/j.envint.2020.105555

67. Zhang H, Zhou X, Sheng N, Cui R, Cui Q, Guo H et al (2018) Subchronic hepatotoxicity effects of 6:2 chlorinated polyfluorinated ether sulfonate (6:2 cl-pfesa), a novel perfluorooctanesulfonate (pfos) alternative, on adult male mice. Environ Sci Technol 52:12809-12818. http://dx.doi.org/10.1021/acs.est.8b04368

\section{Figures}



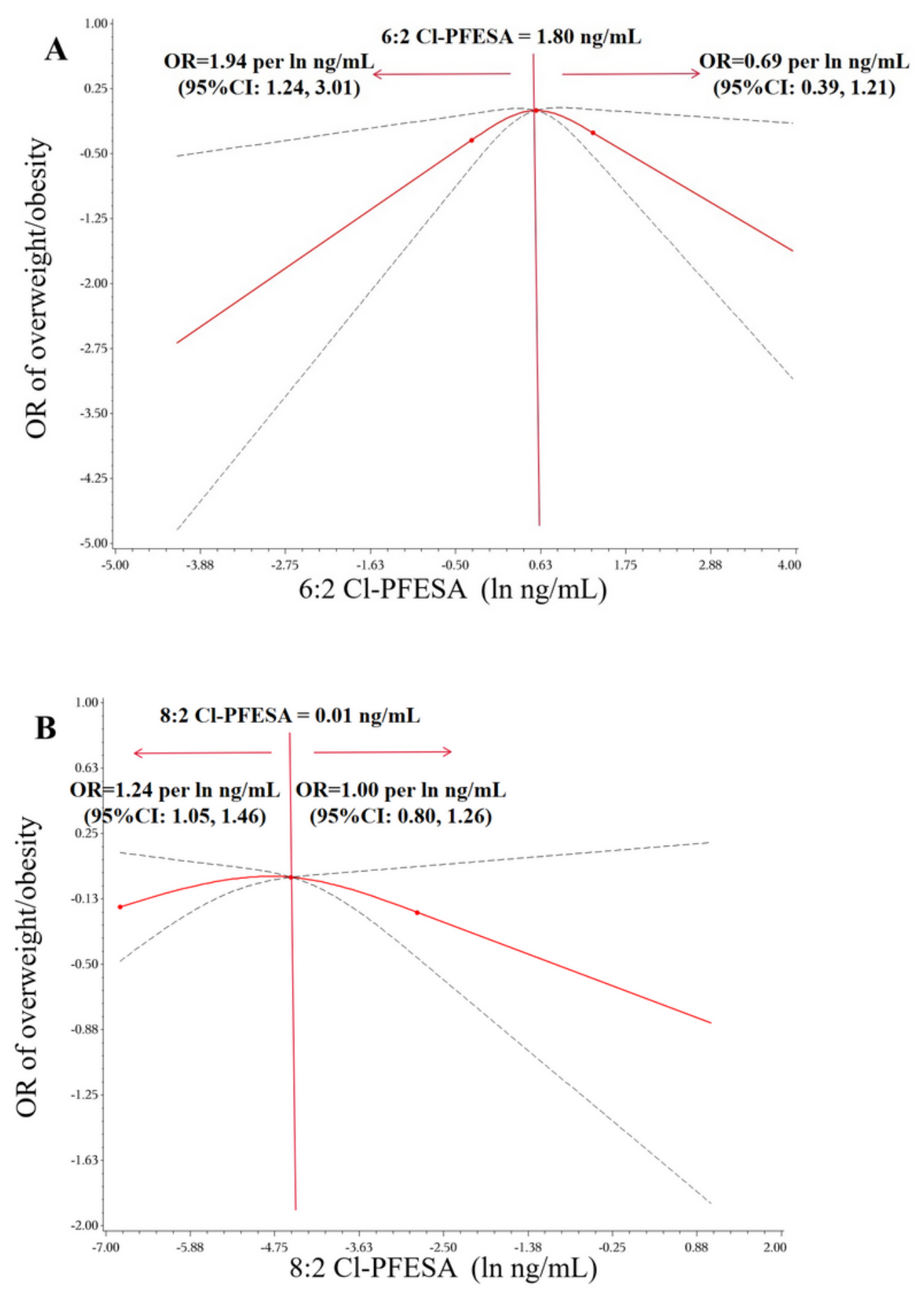

\section{Figure 1}

Dose-response relationship of participants 6:2 Cl-PFESA (A) and 8:2 Cl-PFESA (B) exposure with obesity The solid curves indicate adjusted odds ratios (OR) for overweight/ obesity, with the dashed curves indicate the $95 \%$ confidence intervals derived from restricted cubic spline. The inflection points for $6: 2 \mathrm{Cl}-$ PFESA and 8:2 Cl-PFESA are $1.80 \mathrm{ng} / \mathrm{mL}$ and $0.01 \mathrm{ng} / \mathrm{mL}$, respectively. Models are adjusted for sex, age, education, occupation, family income, smoking, drinking, and physical activity $(n=1275)$ 


\section{Supplementary Files}

This is a list of supplementary files associated with this preprint. Click to download.

- SupplementaryInformation.pdf 